\title{
IRF7 and STAT1 gene expression profile in peripheral blood mononuclear cells of patients with systemic sclerosis
}

\author{
Ramazan Rezaei ${ }^{1}$, Hoda Kavosi ${ }^{2,3}$, Farhad Gharibdoost ${ }^{2}$, Hanieh mojtahedi ${ }^{1}$, Mohammad Vodjgani ${ }^{4 *}$, \\ Mahdi Mahmoudi ${ }^{2,3 *}$
}

${ }^{1}$ Department of Immunology, School of Medicine, Shahid Beheshti University of Medical Sciences, Tehran, Iran. ${ }^{2}$ Rheumatology Research Center, Tehran University of Medical Sciences, Tehran, Iran. ${ }^{3}$ Inflammation Research Center,Tehran University of Medical Sciences, Tehran, Iran. ${ }^{4}$ Department of Immunology, School of Medicine, Tehran University of Medical Sciences, Tehran, Iran.

The critical role of IFN signature genes has increasingly been surveyed to determine the etiology and pathogenesis of systemic sclerosis (SSc). Interferon-regulatory factors (IRFs) and signal transducers and activators of transcription (STATs) are mainly considered as transcriptional modulators of IFN-signature genes and type I interferon and play a major role in the regulation of numerous aspects of an immune response. The current study aimed to assess the transcriptional levels of IRF7 (interferonregulatory factor 7) and STAT1 (signal transducers and activators of transcription 1) mRNAs in PBMCs of scleroderma patients and compare them with those of healthy subjects.

In this study, PBMCs were obtained from 50 scleroderma patients and 30 healthy individuals. Subsequently, total RNA was extracted from isolated PBMCs and cDNA synthesis was carried out. IRF7 and STAT1 mRNA expressions were assessed by applying quantitative real-time PCR, SYBR Green method, and specific primers for IRF7 and STAT1.

Relative expression of IRF7 was significantly increased in the patient group compared with the control group. Moreover, relative expression of IRF7 in limited SSc (1SSc) and diffuse SSc (dSSc) was significantly increased compared with healthy subjects ( $p$ $<0.05$ ). The relative expression of STAT1 transcripts in PBMCs was not statistically significantly different between the patient group and the control group. The correlation between IRF7 expression and the Rodnan score (RS) of the disease was significant. Considering the overexpression of IRF7 in SSc patients and significant correlation between the IRF7 and the Rodnan score of the disease, it is suggested that impaired expression of IRF7 is involved in the pathogenesis of SSc.

Keywords: PBMCs, Gene expression, IRF7, STAT1, Systemic sclerosis

\section{Introduction}

Scleroderma, also called systemic sclerosis (SSc), is a multi-organ rheumatic disorder characterized by widespread skin and internal organ fibrosis, immune dysregulation, and predominant vasculopathy[1]. Based on the extent of affected skin and internal organs, systemic sclerosis is generally divided into two class subsets: diffuse cutaneous SSc (dSSc) and limited cutaneous $\mathrm{SSc}$ (LSSc)[2]. Surprisingly, the involvement of internal organs such as the musculoskeletal system, heart, kidneys, esophagus, and lungs has been reported in both subsets of the disease[3]. Despite broad studies over the past two decades, research has failed to identify the specific etiology and pathogenesis of SSc. Therefore, the main hindrance to the development of an effective treatment for this disease is the inconclusive perception of SSc etiology and pathogenesis[4, 5]. Particularly, comprehensive conception of SSc etiopathogenesis is critical to developing more targeted and effective agents for the treatment of SSc patients.

Oligonucleotide microarray studies on peripheral blood mononuclear cells (PBMCs) of scleroderma patients have demonstrated the significant upregulation of IFN-inducible or IFN signature genes[6]. This pattern of overexpression of IFN signature genes in PBMC of scleroderma patients is

Personal non-commercial use only. Rheumatology Research Journal. Copyright $($ C) 2020. All rights reserved

*Corresponding Author: Mahdi Mahmoudi, Ph.D., Rheumatology Research Center (RRC), Shariati Hospital, Tehran University of Medical Sciences (TUMS), Tehran, Iran. PO-Box: 1411713137, E-mail: mahmoudim@tums.ac.ir, Telefax: +98-218-822-0067.

And Mohammad Vodjgani, PhD, Department of Medical Immunology, School of Medicine, Tehran University of Medical Sciences (TUMS), Tehran, Iran. Email: vojganim@sina.tums.ac.ir.

Received: 04 November 2019; Accepted: 04 January 2020 
represented in CD4+ T-cells and monocytes[7, 8]. Interestingly, IFN-signature genes were overexpressed in both SSc and SLE patients[9]. More than 50\% of the systemic sclerosis subjects had an upregulation of IFNinducible genes[10]. In addition, the effect of genes and genetic variations have been elucidated in different studies[11, 12]. Accordingly, the current study aimed to evaluate the mRNA expression levels of IRF7 (interferonregulatory factor7) and STAT1 (signal transducers and activators of transcription 1) as an interferon signature gene in the PBMCs of scleroderma patients and to compare them with those of healthy subjects. Assessment of the expressions of IRF7 and STAT1 transcripts will assist us in promoting our knowledge about the exact molecular mechanisms of SSc pathogenesis with respect to IFNs signaling pathways, which may result in new therapeutic targets in the future.

\section{Materials and Methods}

\section{Patients and healthy subjects groups}

In this case-control study, PBMCs were collected from fifty SSc patients (25 dSSc and $25 \mathrm{lSSc}$ ) based on the American College of Rheumatology Criteria (ACR) for $\mathrm{SSc}$ identification (Figure. 1). The average disease duration of the SSc patients was $7.8 \pm 2.9$ years. In addition, 30 healthy sex- and age-matched subjects ( 24 females and 6 males, mean age of $42.06 \pm 1.3$ years) were enrolled in this study (Table 1). The healthy subjects had not taken any cytotoxic therapies and did not have a history of skin, rheumatologic, autoimmune, or inflammatory in themselves or among their family members. Samples were taken from scleroderma patients who were recruited from the Rheumatology Center, Shariati Hospital, Tehran, Iran. Informed consent to participate was obtained from all subjects included in the study. The Ethics Committee of Tehran University of Medical Sciences approved all study procedures. The clinical characteristics and demographic information of the participants are represented in Table 1 in more detail.

\section{RNA isolation and cDNA synthesis}

Peripheral blood mononuclear cells were separated using Ficoll-Hypaque density gradient centrifugation protocols. Subsequently, a high pure miRNeasy Mini RNA Extraction Kit (Qiagen, Germany) was applied for total cellular RNA extraction in accordance with the manufacturer's instructions. cDNA synthesis was conducted on $1 \mu \mathrm{g}$ of the extracted RNA using a miScript II RT Kit (Qiagen, Germany). Concisely, $4 \mu \mathrm{L}$ of extracted RNA $(1 \mu \mathrm{g})$ was mixed with $4 \mu \mathrm{L}$ of $5 \mathrm{x}$ miScript HiFlex Buffer, $2 \mu \mathrm{L}$ miScript Reverse Transcriptase Mix, $2 \mu \mathrm{L} 10 \mathrm{x}$ miScript Nucleics Mix, and $12 \mu \mathrm{l}$ RNase- free water for a total volume of $20 \mu \mathrm{L}$. Then, PCR reaction was conducted with conditions of 5 minutes at $95^{\circ} \mathrm{C}, 60$ minutes at $37^{\circ} \mathrm{C}$, and then chilled on ice.

\section{Quantitative real-time PCR analysis}

Primers for IRF7, STAT1, and $\beta 2 \mathrm{M}$-microglobulin $(\beta 2 \mathrm{M})$ as the housekeeping gene were designed from primer blast. The specificity and accuracy of all primers were certified by applying the NCBI Primer-Blast Tool (http://www.ncbi.nlm.nih.gov/tools/primer-blast/). For evaluation of relative expression, quantitative analysis was conducted by real-time PCR applying the StepOnePlus Real-Time PCR System (both Applied Biosystems) and SYBR Green gene expression master mix. Each tube of reaction mixture included $12.5 \mu \mathrm{L} 2 \mathrm{x}$ Taq DNA Polymerase Master Mix RED (Ampliqon, Herlev, Denmark), $3 \mu$ assay mix, $4.5 \mu \mathrm{l}$ cDNA (equivalent to $10 \mathrm{ng}$ of RNA), and $6.5 \mu \mathrm{l}$ $\mathrm{H} 2 \mathrm{O}$ for a total volume of $25 \mu \mathrm{L}$. The qPCR conditions were as follows: 15 minutes at $95{ }^{\circ} \mathrm{C}, 15$ seconds at $95^{\circ} \mathrm{C}$ for 40 cycles, and $60{ }^{\circ} \mathrm{C}$ for 1 minute. The relative expression of $\beta 2 \mathrm{M}$ as the housekeeping gene was applied for normalization. The relative expression of IRF7 and STAT1 in samples from patients and healthy subjects was measured and normalized to the corresponding $\beta 2 \mathrm{M}$ mRNA level using the $2^{-\Delta \Delta \mathrm{CT}}$ method.

\section{Statistical Analysis}

IBM SPSS software (version 21; Chicago, IL, USA) was used for data analyses. IRF7 and STAT1 expression levels are represented as mean $\pm \mathrm{SD}$. Graphs were drawn using GraphPad PRISM 6. In addition, the KolmogorovSmirnov test was used to evaluate the normality of continuous variables. According to the results of the normality test, the Mann-Whitney U test was employed for the statistical assessment of the difference in IRF7 and STAT1 expression levels between the patient and healthy control groups. To investigate the association between two quantitative or continuous variables, such as IRF7 expression, Rodnan Score, ESR, and methylation Pearson Correlation were applied. Pearson's correlation coefficient (r) is a measure of the strength of the association between two variables. P values of $<0.05$ were considered statistically significant.

\section{Results}

\section{Demographic results}

The average disease duration of SSc patients was $7.8 \pm$ 2.9 years. Out of 50 patients, $41(82 \%)$ were female and 9 $(18 \%)$ were male. In the control group, $24(80 \%)$ were female and $6(20 \%)$ were male. The gender distribution between patients and controls was statistically significant ( $p$ value $=0.00036$ and $p$ value $=0.00083$, respectively; Figure $1 \mathrm{~A})$. Considering the ethnicity distribution, the patient and control groups were mixed populations of all Iranian ethnic groups. The frequency distribution of patients and healthy controls based on ethnicity is represented in Figure 1B. In subgroup analysis, a significant difference was found between SSc patients and healthy subjects in Lor and Turk groups ( $p$ value $=0.031$ and $p$ value $=0.042$, respectively)

Relative expressions of IRF7 in PBMCs of SSc patients compared with healthy controls

The relative expression of IRF7 in the peripheral blood mononuclear cells of SSc patients and healthy controls was assessed by quantitative real-time PCR. It was determined 
that the relative expression of IRF7 transcript was significantly increased in the SSc patients compared with the healthy controls $(43.04 \pm 18.3$ vs. $12.28 \pm 7.9$, fold change $3.52, p$ value $=0.0012$; Figure $2 \mathrm{~B}$ ). In addition, subgroup analysis revealed that IRF7 expression in dSSc and 1SSc was considerably enhanced compared with the control group ( $p$ value $=0.0081$ and $p$ value $=0.005$ respectively; Figure $2 \mathrm{~B}$ ). However, the difference in relative expression of IRF7 mRNA between the 1SSc and $\mathrm{dSSc}$ patients did not reach statistical significance.

Relative expressions of STAT1 in patient group compared with healthy control group

The relative expression of STAT1 in the peripheral blood mononuclear cells of SSc patients and healthy controls was assessed by quantitative real-time PCR. It was found that the relative expression of STAT1 transcripts between PBMCs of the scleroderma patients group and the healthy control group was not statistically significantly different (Figure 2A).

\section{Correlation of IRF7 and STAT expression and with clinical manifestations}

To evaluate any possible relations between variables, the correlation coefficient was used. The correlation between IRF7 and STAT1 gene expression missed statistical significance $(\mathrm{r}=0.22, p$ value $=0.12$; Figure $\underline{3 \mathrm{~A}}$ ). In SSc patients, relative expression of IRF7 was found to be significantly correlated with the Rodnan score (RS) of the disease $(\mathrm{r}=0.31, p$ value $=0.034$; Figure $3 \mathrm{~B})$. Indeed, as the expression of the IRF7 gene increased, the severity of clinical manifestations and RS of the disease increased. Moreover, there was a non-significant correlation between the relative expression of STAT1 and the RS of the disease $(\mathrm{r}=-0.213, p$ value $=0.14$; Figure 3C). Furthermore, no significant correlation was demonstrated between the relative expression of IRF7 and erythrocyte sedimentation rates (ESR) in SSc patients $(r=$ $-0.055, p$ value $=0.71$; Figure $3 \mathrm{D})$.
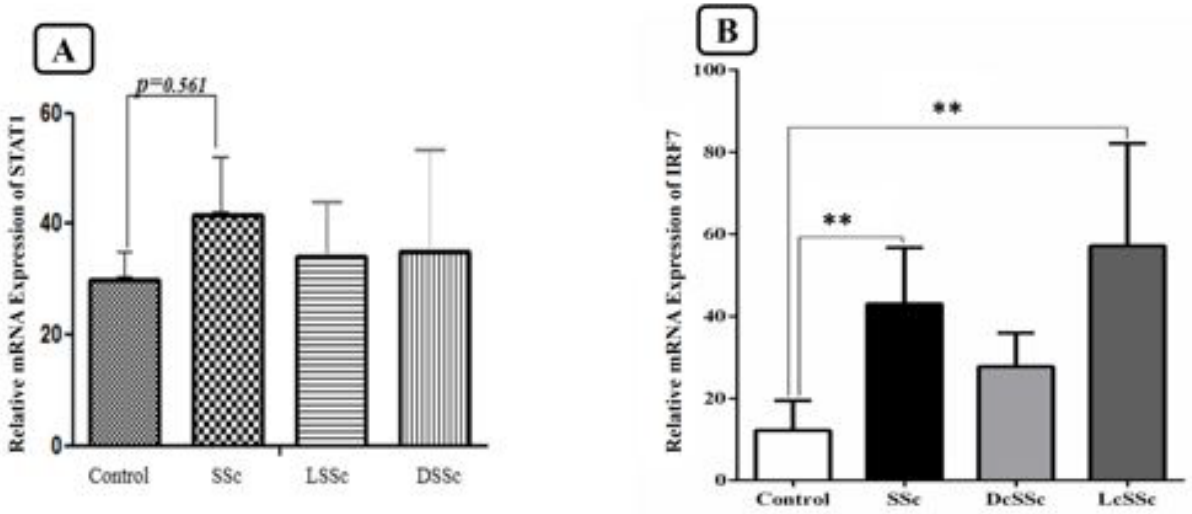

Figure1. Comparison of ethnicity frequency distribution between patient and healthy control groups.
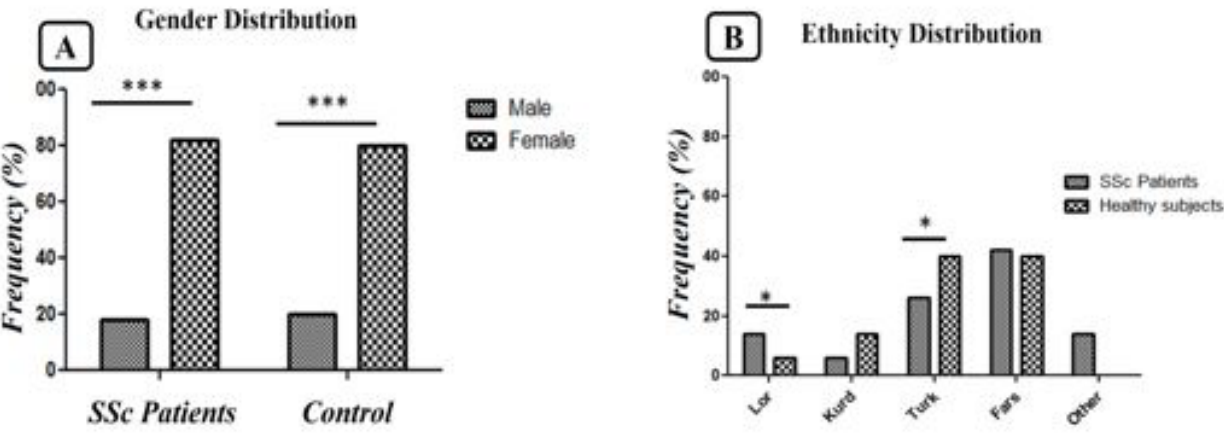

Figure2. Expression of STAT1 and IRF7 in patient and control groups (A). Expression of STAT1 was not statistically significantly different in the patient group compared with the control group. (B). The relative expression of IRF7 in the patient group was significantly enhanced compared with the healthy control group ( $43.04 \pm 18.3$ vs. $12.28 \pm 7.9$, fold change $3.52, p=0.0012)$. In addition, the expression of IRF7 in the dSSc and 1SSc groups was statistically augmented compared with the control group $(p=0.0081$ and $p=$ 0.005 , respectively). However, there was no statistical difference in the expression of IRF7 between the 1SSc and dSSc groups ( $p=$ 0.573). Data is shown as mean $\pm \mathrm{SD}$ 

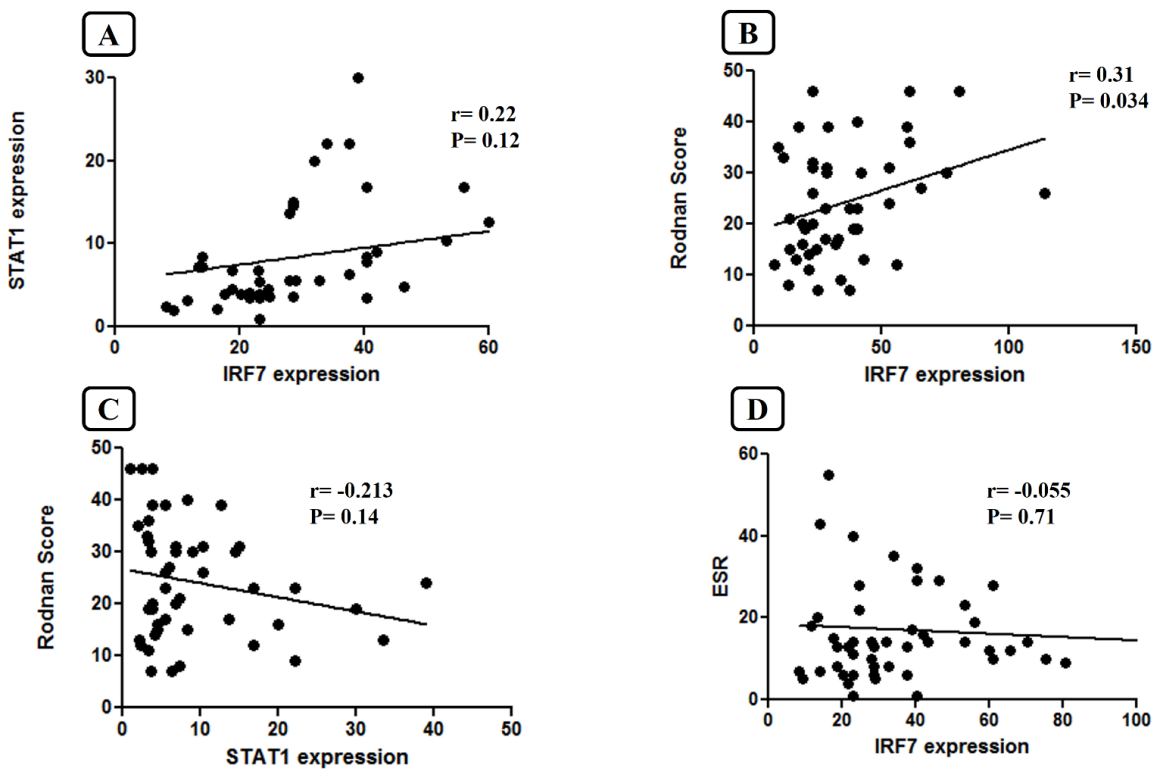

Figure3. Representative of the scatter plot diagram showing the correlation between IRF7 and STAT gene expression (A) and Rodnan disease score (B). In addition, the correlations between STAT1 and the Rodnan score of the disease (C) and IRF7 expression and ESR are shown (D). The regression line is also shown. Pearson correlation coefficient (r) is considered.

Table 1. Baseline and demographic features of SSc patients and healthy controls.

\begin{tabular}{cccc}
\hline & SSc patients $(\mathbf{N}=\mathbf{5 0})$ & Healthy subjects(N $=\mathbf{3 0})$ & $\boldsymbol{p}$ value \\
\hline Gender & $9(18 \%)$ & $6(18 \%)$ & 0.63 \\
Male & $41(82 \%)$ & $24(82 \%)$ & 0.71 \\
Female & $42.84 \pm 1.16$ & $42.06 \pm 1.3$ & 0.65 \\
Age & $22 \pm 3.6$ & $16 \pm 2.9$ & - \\
ESR & $7.8 \pm 2.9$ & & - \\
Disease duration & $21.3 \pm 1.6$ & & - \\
Rodnan score & & & \\
\hline
\end{tabular}

\section{Discussion}

Interferon signature genes include mainly signal transducer and activator transcription (STAT) and interferon regulatory factor (IRFs) families and participate in the regulation of the IFN signaling pathway [12]. The role of type I interferons in the etiopathogenesis of systemic autoimmune disorders including Sjogren's syndrome[13], scleroderma[5], and systemic lupus erythematous [14] has been implicated in the last decade. Type I IFNs (IFN- $\alpha$ and IFN- $\beta$ ) have pleiotropic effects on target cells, inducing self-proliferating pathways, directing numerous immunomodulatory activities, and facilitating antitumor and antiviral activities, with the capacity to promote both innate and adaptive arms of the immune system[15]. Interferon regulatory factors are mainly identified as transcriptional regulators of IFN-signature genes and play an important role in the setting of many aspects of immunity[16]. IRF7 and STAT1, as main transcriptional factors of interferon signaling pathways, have preserved DNA-binding motifs which are found at the N-terminal and identify a consensus oligonucleotide sequence known as the IFN-stimulated response element (ISRE) and GAS (gamma-activated sites) motifs in the promoter sections of target genes[17]. Moreover, ISRE elements which are regulated by IRFs have been demonstrated in many other genes, including the EBV latent membrane oncoprotein 1 (LMP1) [24], p53 as a 
tumor suppressor gene [23], and the transporter associated with antigen processing 2 (Tap2)[18]. As these transcripts are included in apoptosis, regulation of cell cycle, antigen presentation, and tumor inhibition, interferon regulatory factors also have critical roles in the modulation of these pathways in addition to their functions in immune regulation[17].

The complete etiology and pathogenesis of scleroderma remains elusive, and there is no certain cure for this disease. For these reasons, morbidity and mortality rates are excessively high[19]. In microarray studies, the overexpression of IFN signature genes in the peripheral blood mononuclear cells of scleroderma patients has been observed[6]. In accordance with the current investigation, the results revealed that relative expression of IRF7 was increased in the peripheral blood mononuclear cells of scleroderma patients compared with those of the healthy subjects. In addition, IRF7 expression in dSSc and 1SSc patients were significantly augmented compared with the healthy control group. However, the relative expression of STAT1 transcripts in the peripheral blood mononuclear cells of scleroderma patients and healthy subjects did not represent a statistically significant difference.

The RS is a standard outcome index for skin disease in scleroderma and is measured by the summation of skin thickness in 17 various body regions (total score $=51$ ). Therefore, this score is considered as an ideal index for clinical manifestations in SSc patients. The results indicated that the relative expression of IRF7 in the peripheral blood mononuclear cells of scleroderma patients was significantly correlated with the RS of the disease. Indeed, as the expression of the IRF7 gene increased, the severity of clinical manifestations and the RS of the disease increased.

In case-control investigations, various genetic variants of IRF7 have been related with SSc disease, and some of these genetic polymorphisms increase the risk of disease, while others act as SSc moderators[20]. Additionally, other genetic variants of the IFN signaling cascade such as STAT4 and IRF5 loci have been demonstrated to be related with systemic lupus erythematous (SLE) and SSc susceptibility[21, 22]. The overexpression of interferon signature genes such as IRF7 promote inflammatory cascades in dermal fibroblasts through the upregulation of Toll-like receptors; hence, it initiates manifestation of systemic sclerosis-related symptoms[23].

Some limitations of this study should be considered. First, fibroblasts are the main cells of the fibrosis characteristics of scleroderma and other fibrotic diseases. In this study, skin fibroblasts were not examined. In addition, the expression profiles of IRF7 and STAT1 in PBMCs were not compared with skin fibroblasts. Second, systemic sclerosis has multiple susceptibility genes with a complex etiology interacting to trigger the disease in concert with environmental and epigenetic factors. However, this study only addressed the role of genetic factors and highlighted the role of IFN signature genes; other genes and environmental and epigenetic factors were not evaluated. Third, considering the genetic similarities between systemic lupus erythematous and scleroderma, this study did not investigate whether variations within the IRF7 promoter region are also involved in SSc susceptibility and clinical manifestations.

\section{Conclusion}

Overall, considering the overexpression of IRF7 in SSc patients and the significant correlation between IRF7 and the Rodnan score of the disease, it is suggested that impaired expression of IRF7 is involved in the pathogenesis of SSc.

\section{Acknowledgments}

The authors thank the patients and healthy subjects for participating in the study. The Tehran University of Medical Sciences (Grant No. 95-01-30-31316) provided financial support.

\section{Conflict of interest}

None.

\section{References}

1. Elhai M, Meune C, Boubaya M, Avouac J, Hachulla E, Balbir-Gurman A. et al. Mapping and predicting mortality from systemic sclerosis. Ann Rheum Dis 2017; 76(11):1897-05. doi: 10.1136/annrheumdis-2017211448 .
2. Matucci-Cerinic M, Kahaleh B, Wigley FM. Review: evidence that systemic sclerosis is a vascular disease. Arthritis Rheum 2013; 65(8):1953-62. doi: 10.1002/art.37988. 
3. Steen VD, Medsger TA, Jr. Severe organ involvement in systemic sclerosis with diffuse scleroderma. Arthritis Rheum 2000; 43(11):2437-44. doi: 10.1002/15290131(200011)43:11<2437::aid-anr10>3.0.co;2-u.

4. Luo Y, Wang Y, Wang Q, Xiao R, Lu Q. Systemic sclerosis: genetics and epigenetics. J Autoimmun 2013; 41:161-67. doi: 10.1016/j.jaut.2013.01.012.

5. Wu M, Assassi S. The role of type 1 interferon in systemic sclerosis. Front Immunol 2013; 4:266. doi: 10.3389/fimmu.2013.00266.

6. Tan FK, Zhou X, Mayes MD, Gourh P, Guo X, Marcum C. et al. Signatures of differentially regulated interferon gene expression and vasculotrophism in the peripheral blood cells of systemic sclerosis patients. Rheumatology (Oxford) 2006; 45(6):694-02. doi: 10.1093/rheumatology/kei244.

7. York MR, Nagai T, Mangini AJ, Lemaire R, van Seventer JM, Lafyatis R. A macrophage marker, Siglec1 , is increased on circulating monocytes in patients with systemic sclerosis and induced by type I interferons and toll-like receptor agonists. Arthritis Rheum 2007; 56(3):1010-20. doi: 10.1002/art.22382.

8. Duan H, Fleming J, Pritchard DK, Amon LM, Xue J, Arnett HA. et al. Combined analysis of monocyte and lymphocyte messenger RNA expression with serum protein profiles in patients with scleroderma. Arthritis Rheum 2008; 58(5):1465-74. doi: 10.1002/art.23451.

9. Assassi S, Mayes MD. What does global gene expression profiling tell us about the pathogenesis of systemic sclerosis? Curr Opin Rheumatol 2013; 25(6):686-91. doi: 10.1097/01.bor.0000434672.77891.41.

10. Agarwal SK, Tan FK, Arnett FC. Genetics and genomic studies in scleroderma (systemic sclerosis). Rheum Dis Clin North Am 2008; 34(1):17-40; v. doi: 10.1016/j.rdc.2007.10.001

11. Gorlova O, Martin JE, Rueda B, Koeleman BP, Ying J, Teruel M. et al. Identification of novel genetic markers associated with clinical phenotypes of systemic sclerosis through a genome-wide association strategy. PLoS Genet 2011; 7(7):e1002178. doi: 10.1371/journal.pgen.1002178.

12. Mamane Y, Heylbroeck C, Génin P, Algarté M, Servant MJ, LePage C. et al. Interferon regulatory factors: the next generation. Gene 1999; 237(1):1-14. doi: 10.1016/s0378-1119(99)00262-0.

13. Mavragani CP, Crow MK. Activation of the type I interferon pathway in primary Sjogren's syndrome. $\boldsymbol{J}$ Autoimmun 2010; 35(3):225-31. doi: 10.1016/j.jaut.2010.06.012.
14. Rönnblom L, Pascual V. The innate immune system in SLE: type I interferons and dendritic cells. Lupus 2008; 17(5):394-99. doi: 10.1177/0961203308090020.

15. Theofilopoulos AN, Baccala R, Beutler B, Kono DH. Type I interferons (alpha/beta) in immunity and autoimmunity. Annu Rev Immunol 2005; 23:307-36. doi: 10.1146/annurev.immunol.23.021704.115843.

16. Tamura T, Yanai H, Savitsky D, Taniguchi T. The IRF family transcription factors in immunity and oncogenesis. Annu Rev Immunol 2008; 26:535-84. doi: 10.1146/annurev.immunol.26.021607.090400.

17. Ning S, Huye LE, Pagano JS. Regulation of the transcriptional activity of the IRF7 promoter by a pathway independent of interferon signaling. $\boldsymbol{J}$ Biol Chem 2005; 280(13):12262-70. doi: 10.1074/jbc.M404260200.

18. Zhang L, Pagano JS. Interferon regulatory factor 7 mediates activation of Tap-2 by Epstein-Barr virus latent membrane protein 1. J Virol 2001; 75(1):341-50. doi: 10.1128/jvi.75.1.341-350.2001.

19. Al-Dhaher FF, Pope JE, Ouimet JM. Determinants of morbidity and mortality of systemic sclerosis in Canada. Semin Arthritis Rheum 2010; 39(4):269-77. doi: 10.1016/j.semarthrit.2008.06.002.

20. Carmona FD, Gutala R, Simeón CP, Carreira P, OrtegoCenteno N, Vicente-Rabaneda E. et al. Novel identification of the IRF7 region as an anticentromere autoantibody propensity locus in systemic sclerosis. Ann Rheum Dis 2012; 71(1):114-19. doi: 10.1136/annrheumdis-2011-200275.

21. Sharif R, Mayes MD, Tan FK, Gorlova OY, Hummers LK, Shah AA. et al. IRF5 polymorphism predicts prognosis in patients with systemic sclerosis. Ann Rheum Dis 2012; 71(7):1197-02. doi: 10.1136/annrheumdis-2011-200901.

22. Dieudé P, Guedj M, Wipff J, Ruiz B, Hachulla E, Diot E. et al. STAT4 is a genetic risk factor for systemic sclerosis having additive effects with IRF5 on disease susceptibility and related pulmonary fibrosis. Arthritis Rheum 2009; 60(8):2472-79. doi: 10.1002/art.24688.

23. Agarwal SK, Wu M, Livingston CK, Parks DH, Mayes $\mathrm{MD}$, Arnett FC. et al. Toll-like receptor 3 upregulation by type I interferon in healthy and scleroderma dermal fibroblasts. Arthritis Res Ther 2011; 13(1):R3. doi: 10.1186/ar3221. 